\title{
Evaluation qualitative des espèces fourragères présentes dans le département de la Bénoué (Nord Cameroun)
}

\author{
Alain LOABE PAHIMI ${ }^{1}$, Salomon TAAH YAMNDOU ${ }^{1}$, Raphael DAMBA ${ }^{2}$ et \\ Arthur DZEUFACK DJOUMESSI ${ }^{3}$
}

\author{
${ }^{1}$ Chercheur à l'Institut de Recherche Agricole pour le Développement, IRAD Cameroun, BP 415, Garoua, \\ Cameroun. \\ ${ }^{2}$ Etudiant en master écologie à l'Université de Ngaoundéré, BP : 455 Ngaoundéré, Cameroun. \\ ${ }^{3}$ Etudiant en thèse de géographie à l'Université de Yaoundé I, BP : 755 Yaoundé, Cameroun. \\ *Correspondant auteur ; E-mail : alainloabe57@gmail.com ; Tél : (+237)694770822.
}

\section{RESUME}

Les espaces pastoraux subissent des régressions et des conquêtes de par le monde. Ceux-ci occasionnent la diminution assez large de la quantité et de la qualité des fourrages encore disponibles. Cette étude a été réalisée dans le but d'évaluer qualitativement les espèces fourragères présentes au sein du département de la Bénoué. La méthode utilisée pour l'identification des espèces fourragères (herbacées et ligneuses) se base sur les différents entretiens menés auprès des éleveurs de ruminants, par le suivi des bovins, caprins et ovins au pâturage et par l'utilisation des clés d'identification disponibles. Les résultats montrent que les zones de pâturages existantes, diffèrent en qualité floristique et selon la localité où l'on se retrouve. En plus, plusieurs essences fourragères existent et appartiennent à environ 41 espèces. Parmi la vingtaine d'espèces fourragères appréciées par le bétail, 05 ligneuses le sont encore plus (Prosopis africana, Daniella oliveri, Acacia sieberiana, Stereospermum kunthianum et Balanites aegyptica). Il en est de même des 02 herbacées : Acroceras amplectens et Pennisetum pedicellatum. Plus d'une dizaine se retrouve en voie de disparition/menacées de nos jours. Si rien n'est fait, dans quelques années on observera une diminution de la richesse floristique en termes de ressources fourragères dans les différentes localités. Par ailleurs, une étude portant sur les éléments nutritifs et une étude quantitative de ces espèces fourragères rencontrées doivent être menées, pour mieux enserrer les espèces à conserver et les meilleurs espaces offrant de meilleures ressources fourragères.

(C) 2020 International Formulae Group. All rights reserved.

Mots clés : Zones de pâturage, richesse floristique, éleveurs de ruminants, clés d'identifications.

\section{Qualitative assessment of fodder species present in the Bénoué division (North Cameroon)}

\begin{abstract}
Pastoral land (areas) worldwide are undergoing rapid decline as a result of urbanization (development). This often leads to a large decrease in the quantity and quality of available fodder. This study was aimed at evaluating quantitatively the various fodder species present within the Bénoué division. The method used for identification of fodder species (herbaceous and ligneous) was through interviews (surveys), conducted with animal-grazers (breeders) pasturing their flocks (cattle, goats and sheep) in the field and using available keys for
\end{abstract}


fodder for pinpointing. The existing pastoral areas (zones), varies in quality and quantity of flora depending on the location where it is found. Several fodder species exist, belonging to about forty-one (41) classes of species. Among the twenty (20) fodder species best valorized by livestock, five (5) ligneous are most preferred (Prosopis africana, Daniella oliveri, Acacia sieberiana, Stereospermum kunthianum et Balanites aegyptica). As for the herbaceous, same is true for two (2) species, Acroceras amplectens et Pennisetum pedicellatum. More than a dozen species happen to be endangered. Thus, if nothing is done in a few years in order to conserve and preserve these endangered species they might become threatened as a result of gradual reduction in terms of their flora abundance within the different localities. In addition, a study relating to the nutritive elements and a quantitative study of these fodder species met must be undertaken, for better enclosing the species to be preserved and best spaces offering of better fodder resources.

(C) 2020 International Formulae Group. All rights reserved.

Keywords: Pastoral areas, flora abundance, livestock grazers, keys of identifications.

\section{INTRODUCTION}

La qualité de la nutrition est un facteur clé de tout système d'élevage des ruminants qui se veut efficace. Ces derniers doivent recevoir tous les éléments nutritifs diététiques essentiels en quantités optimales. Cependant, plusieurs facteurs sont impliqués et largement responsables des situations de carences tels que : l'intensification d'élevage, l'instabilité du climat, la fertilisation, les caractéristiques du sol qui influencent la biodisponibilité de ces nutriments (Arab et al., 2009). Ces contraintes précarisent l'accès aux ressources pastorales et compromettent davantage la mobilité du bétail (Donou et al., 2008; Kiema et al., 2012). Généralement et de façon actuelle en zones arides et semi-arides, le contexte alimentaire se caractérise chez les ruminants par une offre fourragère insuffisante tant sur le plan qualitatif que quantitatif. Cette insuffissance, couplé à la détérioration de la qualité fourragère s'accompagne d'une baisse de la digestibilité (Nkana et al., 2020). L'alimentation de ces animaux est constituée par une végétation annuelle spontanée des pâturages naturels, des jachères ainsi que par les résidus de l'agriculture, principalement de la paille (Arab et al., 2009).

La raréfaction de l'espace où faire pâturer les animaux et la dégradation des dernières aires de pâturages diminuent largement la quantité et la qualité des fourrages encore disponibles (FAO, 2012). C'est ainsi, qu'en zone soudano-sahélienne et au Cameroun dans l'Arrondissement de Garoua 3 en particulier, l'augmentation des espaces agricoles conduit à une restriction d'espaces pastoraux jusqu'aux limites qui ne sont pas compatibles avec les besoins de déplacement et de nutrition du cheptel. Ces contraintes conjuguées avec l'absence de pratique de la culture fourragère, amènent les éleveurs et les grands agroéleveurs de cette zone à recourir à différentes formes de mobilité (Dugué et al., 2004 ; Dongmo, 2009). Au cours de l'année, une partie du bétail est conduite sur de longs circuits journaliers autour du terroir et l'autre part en transhumance pour valoriser les pâturages et les résidus de cultures. Les différents espaces qu'ils exploitent, s'approprient ou revendiquent et qui sont parfois discutés avec les agriculteurs en général, ne sont plus ou moins que des « territoires d'élevage » dont il est nécessaire de préciser le statut, le fonctionnement et les modes d'usage, afin d'en déterminer les conditions de gestion durable. Cette transhumance est actuellement confrontée à plusieurs contraintes, notamment l'incertitude climatique, les pressions démographiques et les politiques publiques mises en place par les Etats (Kperou et al., 2020). De plus la diversité d'espèces fourragères (ligneuses et herbacées) dans cet arrondissement fait face à des problèmes de conservation et de pérennisation dus à l'extension d'espaces. Dans ce contexte, l'objectif de cette étude est donc de qualifier les espèces fourragères présentes dans l'arrondissement.

\section{MATERIEL ET METHODES Zone d'étude}

L'étude a été conduite pendant trois mois (décembre 2019 à février 2020). 
L'arrondissement de Garoua 3 (Figure 1), de par son caractère fortement rural, avec de vastes espaces, la disponibilité en fourrage, en résidus agricoles et en points d'eaux permanents, demeure sans doute la zone d'étude propice, comparé à Garoua 1 et 2 fortement urbanisées. Quelques villages propres aux éleveurs, ou constituée d'agriculteurs et d'éleveurs dans l'arrondissement de Garoua 3, à savoir : Diambaba, Perma, Douka, Kaidoute, Israël, Djalingo, Lainde bilonde, Sanguere Ngaoundéré, Ouro Tchaka, Kawtal et Sarki yayi, ont donc été choisis pour l'étude. Ce choix était en parfaite adéquation avec le critère de «sélection d'éleveurs disposant de ruminants en nombre élevés, et qui sont plus ou moins amenés à se déplacer selon les périodes de l'année ».

La population se trouvant dans les villages propres aux éleveurs, est constituée de Mbororo ou de Peuhls. Mais celle se trouvant dans les villages comme: Djalingo, Perma, Sanguere Ngaoundéré etc., est généralement combinée à d'autres ethnies comme les Moudangs, les Mboms, les Lackas, les Toupouri, les Guisgas, les Massas, etc. La majeure partie de la population de ces autres villages ne pratique généralement pas l'élevage intensif.

Le relief est essentiellement constitué d'une vaste plaine qui regorge de nombreuses zones marécageuses ou inondées, quelle que soit la période de l'année. Le climat est tropical de type soudanien. Il est caractérisé par une longue saison sèche d'octobre à avril et une courte saison des pluies de mai à septembre. La pluviométrie moyenne annuelle s'élève à 1000 $\mathrm{mm}$ d'eau. Les températures restent élevées avec une moyenne de $28{ }^{\circ} \mathrm{C}$ et des maxima atteignant 40 à $45{ }^{\circ} \mathrm{C}$ en mars et avril (PCD, 2011). Mais depuis quelques années avec les variations de plus en plus importantes du climat, on assiste généralement soit à une augmentation des mois de saison sèche comme de saison des pluies. La végétation est constituée de savane boisée et des galeries forestières à certains endroits, généralement le long des cours d'eau. Les espèces floristiques rencontrées sont diverses et variées. Cependant, la pression causée par l'agriculture, les besoins en bois de chauffage et d'œuvres et la production d'éléments dérivés du bois comme le charbon ont dégradé le paysage qui est devenu plus ou moins arbustif aujourd'hui.

\section{Échantillonnage}

Le choix des enquêtées dans les villages choisis, s'est fait aléatoirement. Vingt-cinq (25) éleveurs par localité pour un total de 275 pour l'ensemble des villages ont été concernés par l'étude. Seuls les éleveurs disposant d'un cheptel de taille variant entre 40 et 100 pour les bovins; entre 10 et 70 pour les ovins et entre 15 et 70 pour les caprins, ont également été concerné. L'administration du questionnaire (conçu grâce au logiciel de collecte de données ODK Collect) a été réalisé en focus groupe et de manière individuelle. Les personnes regroupées disposaient d'un temps de 15 minutes. Pour celles interrogées individuellement, nous avons aménagé un temps pour répondre aux questions de 05 minutes. Cette méthodologie a pour objectif d'apprécier de façon sélective et qualitative, les espèces fourragères auxquelles la population animale a accès.

\section{Identification/Recensement qualitatif des espèces fourragères présentes dans l'Arrondissement}

L'identification des espèces fourragères (herbacées et ligneuses) s'est faite grâce aux différents entretiens menés auprès des éleveurs et par un suivi des bovins, caprins et ovins au pâturage. Toutes les espèces broutées par les animaux ont été recensées. Trois passages ont été fait au cours du suivi des bêtes, afin d'avoir le maximum d'information sur les espèces broutées et appétées. Aidé également du document de Malzy (1954) sur les graminées $\mathrm{du}$ Nord Cameroun et leurs utilisations; la fiche technique de Gautier et al. (2002); le guide des adventices d'Afrique de l'ouest de Okezie et al. (1989); le manuel Arbres, Arbustes et Lianes des zones sèches d'Afrique de l'Ouest de Michel Arbonnier (2000), nous avons procéder à l'indentification des espèces. 


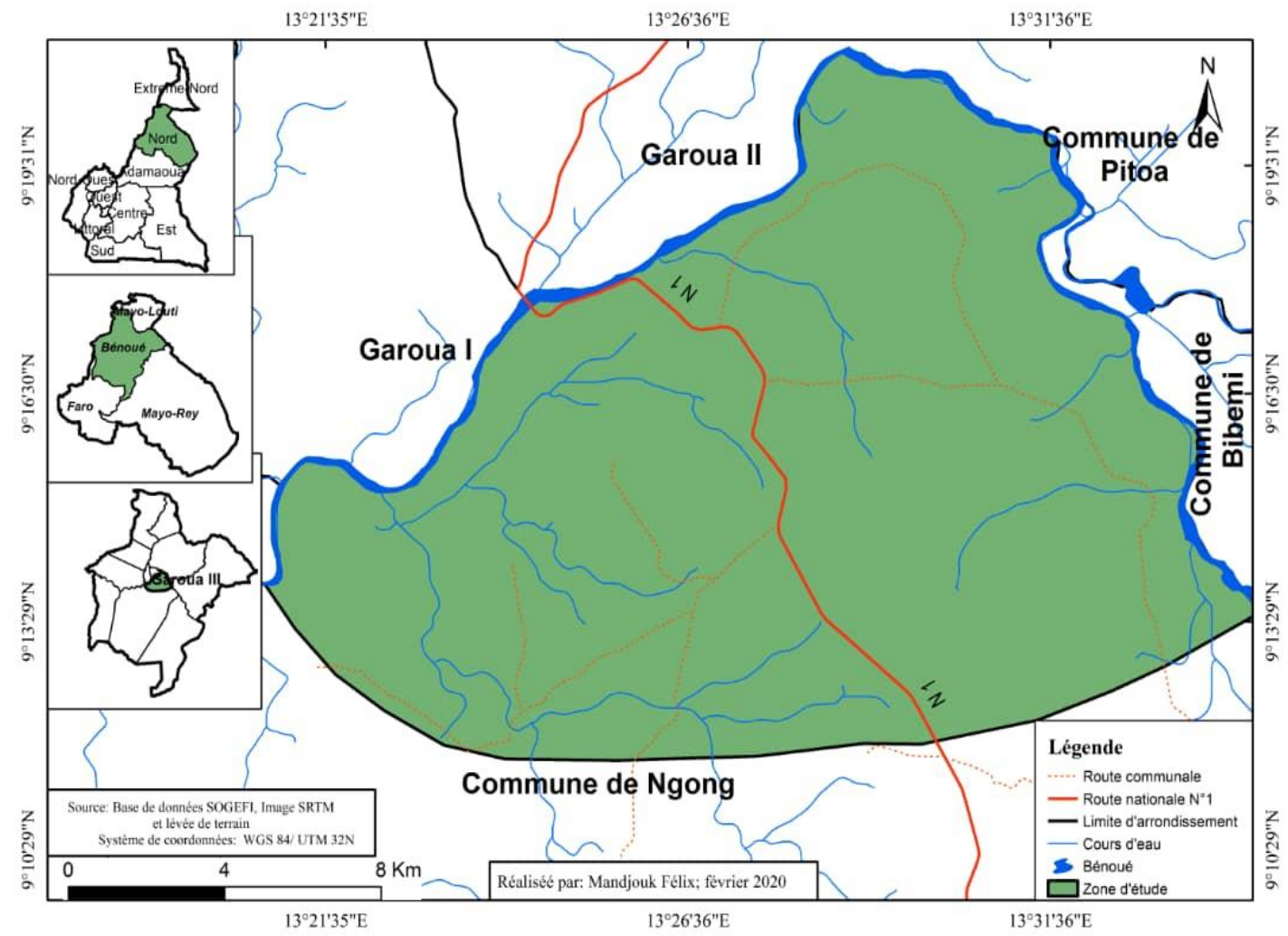

Figure 1 : Carte de localisation de la zone d'étude.

\section{RESULTATS}

\section{Espaces de pâturage actuel et période de déplacement en zone de parcours}

Les zones de pâturages, diffèrent selon la localité/village où l'on se retrouve : les éleveurs se trouvant dans les localités enquêtées, ont chacun à ce qui leurs concerne une préférence en zone de parcours. Ils préfèrent pour certains les espaces de pâturages de la zone de Lainde - Les espaces agricoles de l'Institut de Recherche Agricole pour le Développement (IRAD), à cause de la présence des résidus agricoles après récoltes - les zones inondables de BOCKLE, à cause de la disponibilité de certaines espèces fourragères appétés, qu'ils exploitent en période sèche lors de leur déplacement de décembre jusqu'en début juin; les abords de la Bénoué au lieu dénommé Domayo, qui est une zone humide, lors de leur déplacement de mars à fin mai ; la zone de Karewa en début novembre, jusqu'en fin mars ; les montagnes non utilisées à des fins agricoles de Sanguéré Paul et de ses alentours pour limiter les conflits «agriculteurséleveurs » à partir de février ; à Sanguéré Ngal, ils préfèrent plus les espaces agricoles qu'ils exploitent en période sèche, généralement en début janvier; Le long du fleuve Bénoué à partir de décembre jusqu'en fin juin; les espaces agricoles de Babla après récolte en début janvier ; les espaces de Beebal pendant la saison pluvieuse de fin juin à début septembre ; à Babagin, Moudjongue, Harande, Hassoumi, et en direction de Perma en début février. Les espaces agricoles de Babla après récolte, de décembre à début juin. Généralement la pâture est menée sur place pour certains éleveurs (Douka, Israël). Pour ces éleveurs, la période de déplacement n'est pas un élément important car ils ne se déplacent presque même pas pour la grande majorité. Le troupeau s'il y a nécessité est conduit au niveau des villages limitrophes. D'autres encore préfèrent les espaces se situant le long de la Bénoué, au niveau du Mayo Boukki (devant Ngong) de février à fin mai. Les Figures 2 et 3 illustrent le 
parcours des animaux en période sèche et en période pluvieuse dans les localités d'étude.

\section{Espèces présentes (herbacées et ligneuses)}

Les espèces fourragères que l'on retrouve actuellement dans l'arrondissement sont variées. Mais on observe de nos jours une disparition d'un nombre élevé d'espèces que l'on observait avant et que l'on ne retrouve plus assez aujourd'hui. Les Tableaux 1 et 2 présentent ces espèces, leurs familles d'origine et leurs noms scientifiques. Ces tableaux montrent qu'une partie seulement de ces espèces est appréciées (espèces ayant au moins un symbole * après son nom scientifique).

\section{Espèces fourragères en voie de disparition/menacées}

Le Tableau 3 présente les fourrages en voie de disparition/menacés au sein du département de la Bénoué. Il ressort de ce tableau, qu'une bonne partie des espèces les plus appréciées par le bétail se trouve être en voie de disparition/menacées.
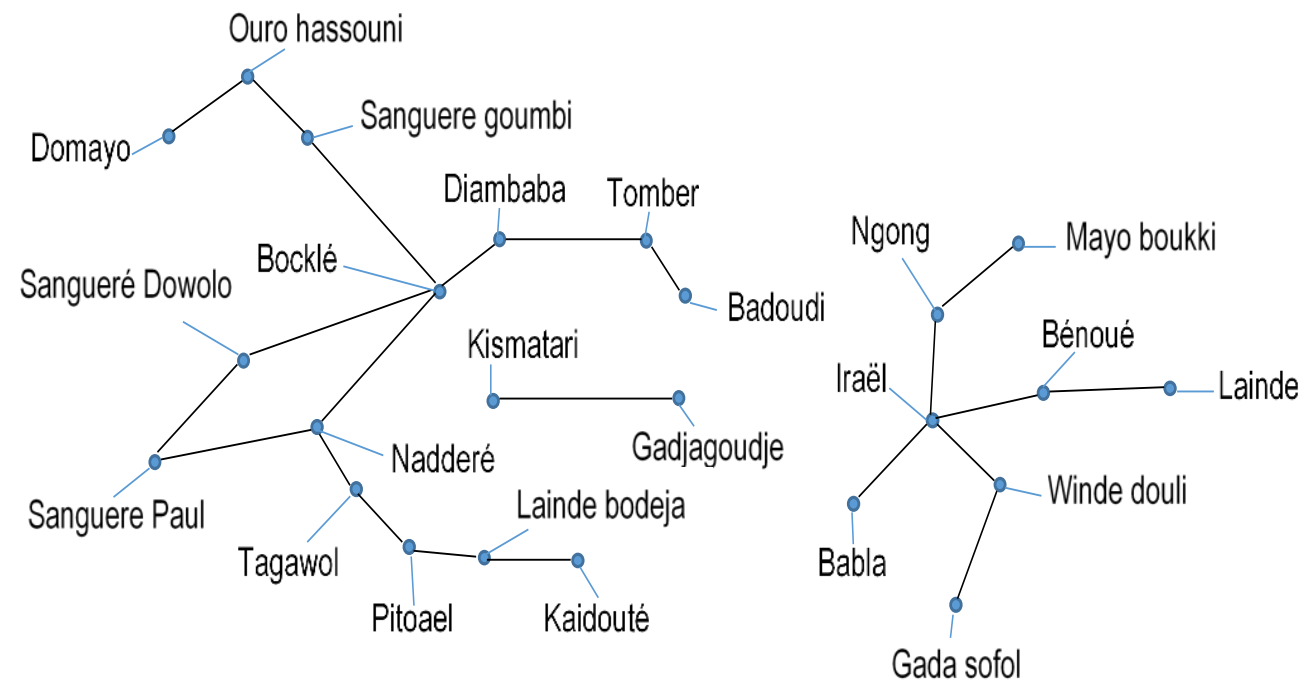

Figure 2 : Parcours des animaux en période sèche en fonction des localités d'étudiées.

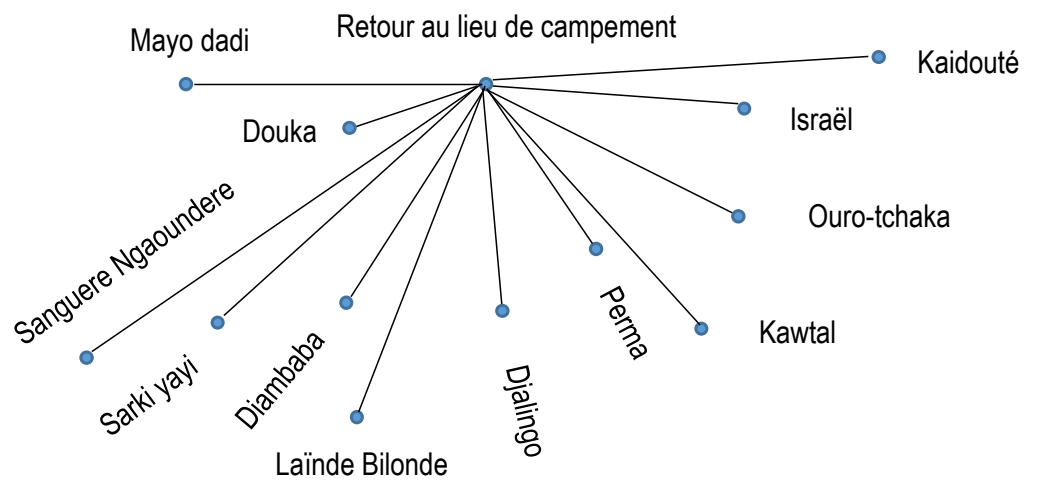

Figure 3 : Déplacement des animaux en saison pluvieuse en fonction des localités d'étude. 
Tableau 1 : Les espèces ligneuses présentent, leurs noms scientifiques et leurs familles.

\begin{tabular}{|c|c|}
\hline Noms Scientifiques & Familles \\
\hline Acacia sieberiana ${ }^{* \% *}$ & Fabacées \\
\hline Acacia senegal & Fabacées \\
\hline Acacia seyal & Fabacées \\
\hline Acacia hockii & Fabacées \\
\hline Acacia nilotica & Fabacées \\
\hline Acacia polycantha & Fabacées \\
\hline Adansonia digitata & Bombacaceae \\
\hline Anogeisus leicarpus* & Combretaceae \\
\hline Balanites aegyptica *** & Zygophyllaceae \\
\hline Conbretum nigricans & Combretaceae \\
\hline Daniellia oliveri $^{* * * *}$ & Fabaceae \\
\hline Faidherbia albida & Fabaceae \\
\hline Ficus platyphylla & Moraceae \\
\hline Ficus sycomorus * & Moraceae \\
\hline Haematostaphis barteri & Anacardiaceae \\
\hline Isoberlinia doka & Fabaceae \\
\hline Khaya senegalensis & Meliaceae \\
\hline Mimosa pigra & Fabaceae \\
\hline Parkea biglobosa & Fabaceae \\
\hline Prosopis senegalensis & Fabaceae \\
\hline Prosopis africana ${ }^{* * * * *}$ & Fabaceae \\
\hline Pterocarpus erinaceus * & Fabaceae \\
\hline Entada africana* & Mimosaceae \\
\hline Sclerocarya birrea & Anacardiaceae \\
\hline Stereospermum kunthianum ${ }^{* * *}$ & Bignoniaceae \\
\hline Sterculia setigera & Malvaceae \\
\hline Terminalia indica & Combretaceae \\
\hline Terminalia avicennioides & Combretaceae \\
\hline Vitellaria paradoxa* & Sapotaceae \\
\hline Ziziphus manritiana * & Rhamnaceae \\
\hline Pseudocedrela kotschyi* & Meliaceae \\
\hline
\end{tabular}

Les espèces qui ont devant leur nom le symbole* sont les plus appréciés. Plus le nombre d'étoile ***** est élevé, plus l'espèce est appréciée comparer aux autres.

Tableau 2 : Les espèces herbacées présentes, leurs noms scientifiques et leurs familles.

\begin{tabular}{|c|c|}
\hline Nom scientifique & Famille \\
\hline Pennisetum pedicellatum ** & Poaceae \\
\hline Acroceras amplectens $* * *$ & Poaceae \\
\hline Paspalidium $s^{*}{ }^{*}$ & Poaceae \\
\hline Leptadenia spartium & Apocynaceae \\
\hline Mimosa pigra & Fabaceae \\
\hline Commelina forsskalaei & Commelinaceae \\
\hline Eulesine indica * & Poaceae \\
\hline Bracharia sp & Poaceae \\
\hline
\end{tabular}




\begin{tabular}{ll}
\hline Penicetum compressum $^{*}$ & Poaceae \\
\hline Perotis indica & Poaceae \\
\hline Echinochloa stagnina $^{*}$ & Poaceae \\
\hline Physolis angulata $^{*}$ & Solanaceae \\
Saccolepis interrupta* & Cyperaceae \\
\hline
\end{tabular}

Les espèces qui ont devant leur nom le symbole* sont les plus appréciés. Plus le nombre d'étoile* * * est élevé, plus l'espèce est appréciée comparer aux autres.

Tableau 3 : Les fourrages en voie de disparition/menacés.

\begin{tabular}{ll}
\hline Ligneuses & Herbacées \\
\hline Acacia seyal & \\
\hline Acacia ataxacantha & \\
\hline Balanites aegyptiaca & \\
\hline Diopyros mespiliformi & Eulesine indica \\
\hline Khaya senegalensis & \\
\hline Parkia viglovosa & Polygonum acuminatum \\
\hline Prosopis africana & \\
\hline Pterocarpus erinaceus & \\
\hline Stereospermum kunthianum & \\
\hline Vitellaria paradoxa & \\
\hline Vitex doniana
\end{tabular}

\section{DISCUSSION}

Les espaces de pâture actuelle sont extrêmement réduits au point où le bétail éprouve de sérieux problèmes pour se déplacer. Il se pose de ce fait un réel problème d'alimentation en période sèche et singulièrement en période pluvieuse. Le bétail ne dispose plus d'assez d'espaces de pâturage, changés en espaces agricoles. C'est en réalité l'une des raisons qui poussent les éleveurs à préférer sans en avoir un éventail de solutions les espaces existants le long des cours d'eaux, des flancs de montagnes, de plus en plus les espaces agricoles pourvus de résidus de culture en période sèche et même à ne pas se déplacer. Ces résultats trouvés corroborent ceux de Moha (2008), Dongmo et al. (2009), Coulibaly et al. (2009) et Bode (2017), et Lesse et al. (2015).

Le véritable constat fait sur le terrain révèle une timidité, voir une socialisation (refus de s'ouvrir entièrement aux autres) obtus des éleveurs (Peuls et Mbororo surtout), qui pourtant participent quand même sur le plan socio-économique, socio-politique et culturelle aux activités de la société. Mais ils n'ont pas pensé à faire reconnaitre leurs espaces de pâturage dominés ou occupés aujourd'hui par les espaces agricoles (constat également fait par Dongmo et al. (2009)). Ils se sont plus attardés à faire reconnaitre leurs différents lieux de résidence, construit de plus en plus de nos jours en matériaux semi-définitif. Aujourd'hui il se pose inévitablement un problème qui se veut grandissant entre éleveurs et agriculteurs, dû à l'empiétement des bêtes sur les espaces devenus majoritairement agricoles (constat fait par Bode (2011)). Au sein de certaines localités enquêtées (Perma, Douka, Israël), le déplacement pour les zones de pâturage, n'est pas forcement considéré comme obligatoire. A Douka et à Israël, localités éloignées du centre de l'arrondissement de Garoua $3^{\text {ème }}$, le problème opposant les agriculteurs et les éleveurs pour le foncier n'est pas encore d'actualité et on observe de vaste espèce servant généralement de zone de parcours et contribuant ainsi à la limitation du déplacement des éleveurs. 
La qualité des espèces fourragères rencontrées au sein des différentes localités démontre effectivement que malgré un rétrécissement des espaces de pâturages, la persistance de certaines espèces herbacées et ligneuses, justifie l'intérêt qu'il faut apporter pour une contribution efficace à la conservation de ces espèces. Mais toutes ces essences ne sont pas appréciées de la même manière par le bétail, certains sont plus appréciées que d'autres et généralement sont fonction de la disponibilité et de la répartition spatiale en fonction des localités. Prosopis africana, Daniella oliveri, Acacia sieberiana, Stereospermum kunthianum et Balanites aegyptica, chez les ligneux; Acroceras amplectens, Pennisetum pedicellatum, chez les herbacées. Cette préférence pouvant ce justifié par la composition en éléments nutritifs importante et recherchés par les animaux dans le fourrage sélectionné.

Avec les différents effets causés par les variations climatiques, la démographie galopante, le rétrécissement des espaces de pâturages, les mauvaises pratiques comme la coupe abusive du bois et les feux de brousses tardifs, certaines essences fourragères tendent à disparaitre et à être considérées comme espèces menacées. Si rien n'est fait dans quelques années, on observera une diminution de la richesse floristique en termes de ressource fourragère. Il faut dans ce cas mener une très forte politique sous forme de réforme foncière dès maintenant en vue de pouvoir dégager les responsabilités des différents acteurs impliqués (gouvernements, éleveurs, agriculteurs, société civile), pour une amélioration de la conservation/préservation du potentiel fourrager disponible (herbacées et ligneuses) au sein de l'arrondissement et même de la région du Nord.

\section{Conclusion}

La présente étude menée sur les essences floristiques et fourragères présentes dans l'arrondissement de Garoua $3^{\text {ème }}$, qui avait pour principale objectif de quantifier les espèces fourragères, a permis d'avoir un idée plus nette sur les essences retrouvées. Une panoplie de ces espèces existe et a été recensée.
Une variété d'espèces ligneuses, ainsi que des herbacées en voie de disparitions/menacées, ont également été recensées. Mais au cours de nos investigations le nombre d'espèces ligneuses en voie de disparition était considérable comparé aux espèces herbacées. Le véritable problème dans cette zone d'étude bien que couplé à l'espace pastoral existant, reste le phénomène crucial de gestion des conflits entre «agriculteurs et éleveurs" auxquels on associe la non conservation et le manque de gestion des espèces fourragères. Une étude des éléments nutritifs et une étude quantitative de ces espèces fourragères rencontrées doivent être menées, pour mieux enserrer les espèces à conserver et les bons espaces offrant de meilleures ressources fourragères, comparé aux autres.

\section{CONFLIT D'INTERETS}

Les auteurs de ce manuscrit déclarent qu'il n'existe aucun conflit d'intérêts entre eux.

\section{CONTRIBUTIONS DES AUTEURS}

Cette étude a été possible grâce à la contribution de tous ses auteurs. En effet, ALP a, non seulement élaboré le protocole de recherche, réalisé la collecte, l'analyse et le traitement des données mais aussi a rédigé la première version du manuscrit. STY a contribué à la collecte, l'analyse des données et à la relecture du manuscrit. RD a participé à la collecte des données et à la relecture du manuscrit. Enfin, quant à $\mathrm{ADJ}$, il a participé à l'élaboration du protocole de recherche et à la relecture du manuscrit.

\section{REMERCIEMENTS}

Les auteurs remercient énormément les chefs des localités enquêtés, pour leurs disponibilités et pour les facilitées accorder lors des différentes descentes de terrain.

\section{REFERENCES}

Arab H, Haddi ML, Mehennaoui S. 2009. Evaluation de la valeur nutritive par la composition chimique des principaux fourrages des zones aride et semi-aride en Algérie. Sciences \& Technologie. C, Biotechnologies, 30 : 50-58. 
Bode S. 2011. Mobilité et mutation : Cas de la communauté de pasteur Wadaabe du lignage des Suudu Suka'el de Tanout (Niger Centre-Est), thèse unique de doctorat en géographie, Département de géographie, Université Abdou Moumouni de Niamey, Niger, p.337.

Bode S. 2017. Dynamiques d'occupation et de valorisation des espaces pastoraux dans le département de Madaoua (Région de Tahoua/Niger). Annales de l'Université de Moundou, Série A - Faculté des Lettres, Arts et Sciences Humaines, 3(1).

Coulibaly D, Poccard Chapuis R, Ba A. 2009. Dynamiques territoriales et changements des modes de gestion des ressources pastorales au Mali Sud (Mali). Renc. Rech. Ruminants, 16 : 357-360.

Gautier D, Hautdidier B, Ntoupka M, Onana J, Perrot N, Tapsou T. 2002. Fiches techniques des Arbres utiles aux paysans du Nord Cameroun. Caractéristiques de l'arbre, ce qu'en font les paysans et ce qu'ils pourraient en faire. $125 \mathrm{p}$. <hal00837556>.

Dongmo AL, Vall E, Liba'A NK, Bechir B, Lossouarn J. 2009. Le territoire d'élevage : diversité, complexité et gestion durable en Afrique soudano-sahélienne. Cas du Nord-Cameroun, Ouest Burkina Faso, Mali-Sud et Sud-Tchad. Actes du colloque « Savanes africaines en développement: innover pour durer ». Garoua, Cameroun. Cirad, 9 p. <cirad00471878v1>.

Donou B, Ogouwalé E, Yabi I, Boko M. 2008. Contraintes climatiques et pression sur les pâturages dans le Département des collines (République du Bénin). Revue de Géographie du Bénin, Université de d'Abomey-calavi (Bénin), 3 : 61-75.

FAO. 2012. La transhumance transfrontalière en Afrique de l'Ouest, Proposition de plan d'action. http://www.interreseaux.org. Consulté le 05 février 2015.

Kiema A, Sawadogo I, Ouédraogo T, Nianogo AJ. 2012. Stratégies d'exploitation du fourrage par les éleveurs de la zone sahélienne du Burkina Faso. Int. J. Biol.
Chem. Sci., 6(4) :1492-1505. DOI : http://dx.doi.org/10.4314/ijbcs.v6i4.8.

Kperou gado BO, Toko imorou I, Arouna O, Oumarou M. 2020. Caractérisation des parcours de transhumance à la périphérie de la réserve de biosphère transfrontalière du W au Bénin. Int. J. Biol. Chem. Sci., 14(2): $\quad 333-352 . \quad$ DOI : https//dx.doi.org/10.4314/ijbcs.v14i2.3.

Lesse P, Houinato MRB, Djenontin J, Dossa H, Yabi B, Toko I, Tente B, Sinsin B. 2015. Transhumance en République du Bénin : états des lieux et contrantes. Int. J. Bio. Chem. Sci., 9(5) : 2668-2681. DOI : https//dx.doi.org/10.4314/ijbcs.v9i5.37.

Malzy P. 1954. Quelques Plantes du Nord Cameroun et leurs utilisations. Journal d'Agriculture Tropicale et de Botanique Appliquée, 1(5-6): 148-179. DOI : 10.3406/jatba.1954.2147.

Michel A. 2000. Arbres, Arbustes et Lianes des Zones Sèches d'Afrique de l'Ouest. CIRAD, UICN, MNHN. Guide de reconnaissance des espèces ligneuses $549 \mathrm{p}$.

Moha M. 2008. Les relations entre agriculteurs et éleveurs en contexte de crise alimentaire à Roumbou-Sakabal. Afrique Contemporaine, 225 : 137-159. DOI: 10.3917/afco.225.0137

Nkana JGK, Mweugang NN, Germanus SB, Semi YA, Ntsafack P, Awantu C, Wangba CT, Noumbissi FGT, Miégoué E. 2020. Effet de quelques légumineuses fourragères sur la reproduction, la croissance et la carcasse chez les cochons d'indes (Cavia porcellus L.). Int. J. Biol. Chem. Sci., 14(2): 600-612. DOI : https//dx.doi.org/10.4314/ijbcs.v14i2.23.

Okezie L, Akobundu, Agyakwa C W. 1989. Guide des Adventices d'Afrique de l'Ouest. Institut International d'Agriculture Tropicale: Ibadan, Nigeria ; 524p.

Plan Communal de Développement (PCD). 2011. Commune de Garoua $3^{\text {ème }}$. Planification communale avec l'appui du Programme National de Développement Participatif (PNDP). TerDel. 211p. 